\title{
Ultrasound guided 4 in 1 block for managing postoperative pain in arthroscopic knee surgery
}

\author{
Parthasarathy Srinivasan $^{1^{*}}$, Harith Dagupatti ${ }^{2}$, Balachander Sarvanan ${ }^{3}$ \\ Professor $^{{ }^{*}}$, Post Graduate Student ${ }^{2}$, Senior Resident ${ }^{3}$, Mahatma Gandhi Medical College and \\ Research Institute, Sri Balaji Vidyapeeth University, Puducherry, 607402, India
}

\begin{abstract}
Surgeries of the knee are usually painful and managing postoperative pain is challenging. Various methods of analgesia are available. Epidural is considered as gold standard but after the advent of ultrasound, peripheral nerve blockade of the lower limb is gaining importance. A 50-year-old male patient posted for arthroscopic knee surgery, completed under spinal anaesthesia, was given four in one block using ultrasound. The point of entry of the needle was in lower thigh where the femoral artery dips down deep. $30 \mathrm{ml}$ of $0.25 \%$ bupivacaine was given in the space above it. Postoperatively, the time of first systemic analgesia was noted which was eight hours after full regression from spinal. Peripheral nerve blocks of the lower limb for knee surgeries usually target only the femoral component. Addressing the sciatic component with a single injection is a real challenge and we did it to block the four nerves with a single point injection. We conclude 4 in 1 block is useful as a postoperative analgesic technique in knee surgeries.
\end{abstract}

Key words: knee pain; postoperative; nerve block; four in one

\section{Introduction}

Knee arthroscopy is one of the commonly performed orthopedic procedures with significant number of patients having moderate to severe pain affecting activity level and satisfaction. ${ }^{1}$ Analgesia after knee surgery can be provided by multiple nonsystemic non-opioid based methods, including local anaesthetic infiltration, peripheral nerve blockade, neuraxial procedures, and intra-articular injections. Relieving pain without compromising motor function is a challenge in early postoperative analgesia. In an effort to provide an effective, safe and long-lasting post-arthroscopy analgesia, intra-articular administration of local anaesthetics has been widely used. ${ }^{2,3}$

*Correspondence: Parthasarathy Srinivasan E mail: painfreepartha@gmail.com

https://orcid.org/0000-0002-3808-6722 Received: 30/08/2018

Accepted:05/11/2018

DOI: http:/doi.org/10.4038/slja.v27i1.8376

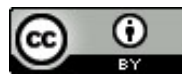

Later epidural technique was used as a method of treating postoperative analgesia. ${ }^{4}$ but patients who receive epidural analgesia may develop autonomic disturbances, unintended motor blockade, urinary retention. ${ }^{5}$ With the advent of ultrasound, the popularity of peripheral nerve blockade has increased. Various studies have confirmed early recovery with adductor canal block over femoral nerve block, with motor sparing effect for knee surgeries. The combination of the femoral nerve block with sciatic nerve block has provided adequate analgesia with lower consumption of perioperative opioids and rescue analgesia, for knee and below knee surgeries. ${ }^{6}$ The superior efficacy of the combined adductor canal block with the sciatic nerve block comes with associated technical difficulties including positioning of patients differently for both the blocks. ${ }^{7}$ Here we describe a case report where a patient underwent knee arthroscopy and the postoperative pain was successfully managed with 4-in-1 block through a single injection point.

\section{Case Report}

A 50-year-old male ASA I patient with knee pain diagnosed as having Left Knee Loose Bodies was posted for knee arthroscopy. His preoperative work up was unremarkable. The 
patient received subarachnoid block with 2.8 $\mathrm{ml} 0.5 \%$ heavy bupivacaine in the left lateral position. The intraoperative period was uneventful. Postoperatively in the recovery room, the patient was kept in the supine position with the ipsilateral leg kept in external rotation, slight abduction and knees slightly flexed. The femoral artery was traced down with a Sonosite Xporte from upper thigh to find the location where it dips down posterior to become less visible. The transducer was kept just above this point and $30 \mathrm{ml}$ of $0.25 \%$ bupivacaine was injected to spread around the artery and also in a plane to push the sartorius up. (Figure 1)

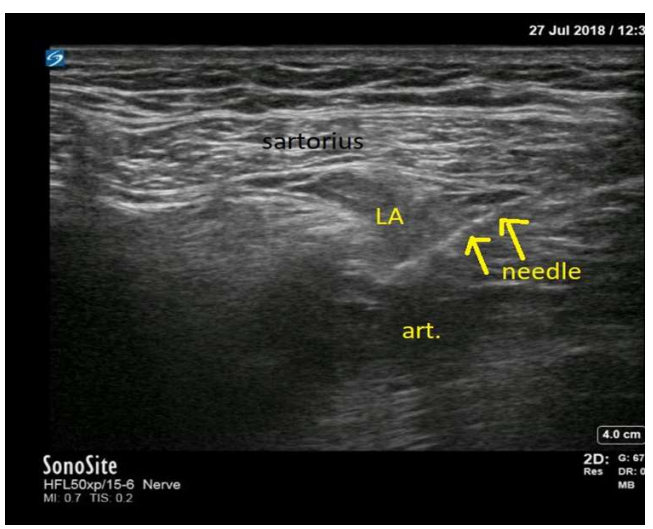

Figure 1: Technique of four in one block

Once the patient had completely recovered from spinal anesthesia in the other lower limb, he had persistent loss of sensation and analgesia in the ipsilateral leg of both the sciatic and femoral components. This confirmed our successful block. We found out that with a single point injection in the supine position, the sciatic nerve was also blocked which we confirmed with ultrasound (Figure 2) The time of first systemically administered analgesia was eight hours after the complete regression of spinal anaesthesia. The VAS pain score remained below 2 for eight hours. Later the postoperative analgesia was managed with oral tramadol and diclofenac.

\section{Discussion}

Peripheral nerve blocks have been proven to be superior to central neuraxial blocks with better postoperative analgesia with fewer side effects, better patient satisfaction and rehabilitation. ${ }^{5}$ The knee is supplied by genicular branches from the nerve to sciatic

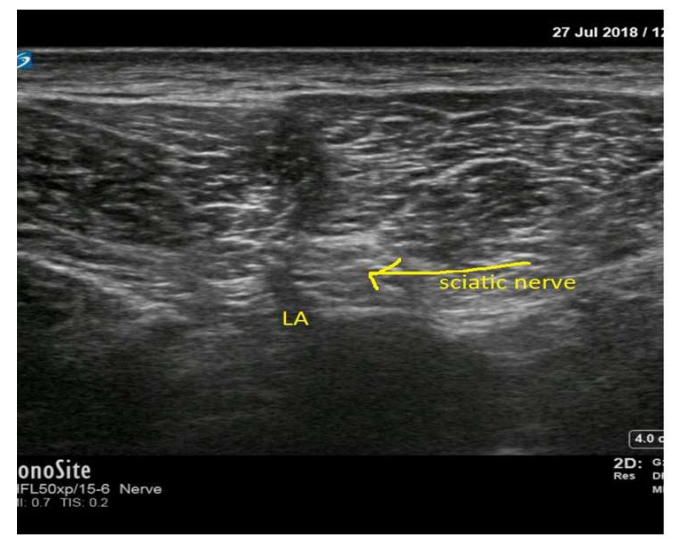

Figure 2: Local anaesthetic around sciatic nerve

nerve: the four targets with single injection. The skin around the knee is supplied by the cutaneous branches from the femoral nerve and the saphenous nerve. The nerve supply of the leg and foot is from the sciatic nerve, except the skin in the medial aspect which is supplied by the sensory saphenous nerve. Suma et $\mathrm{al}^{9}$ compared the continuous femoral nerve blockade versus epidural analgesia for postoperative pain relief in knee surgeries and concluded that both are effective with former having fewer side effects. Many studies have concluded that the combination of sciatic with femoral nerve block improves postoperative analgesia significantly. The analgesia for knee and below knee surgeries can be achieved by combining adductor canal block and sciatic nerve block. ${ }^{10}$ Combining these two blocks needs change of position and logistic difficulties in trauma patients. A single injection technique to block all these nerves can provide ease of practice and better postoperative care analgesia

Ritish Roy et al ${ }^{8}$ concluded that the positional and technical difficulties with multiple blocks can be overcome by using a single injection 4-in-1 block technique. Similar to this study, we have demonstrated the spread of the drug to the sciatic nerve from the adductor canal. Hence this block can be performed easily to target sciatic nerve and effect complete analgesia after knee surgeries. This case report is presented for its novelty and absence of technical difficulties.

\section{Conclusion}

Four in one nerve block leading to blocking all the nerves around the knee is easy and 
safe: can be done in supine position with single injection technique. It is one of the easily administrable nerve blocks to achieve analgesia of the sciatic nerve distribution in the supine position.

\section{References}

1. Drosos G, Stavropoulos N, Katsis A, et al. Post-Operative Pain After Knee Arthroscopy and Related Factors. Open Orthop J. 2008;13:110-4.

https://doi.org/10.2174/1874325000802010110

2. Das A, Majumdar S, Kundu R, Mitra T, et al. Pain relief in day care arthroscopic knee surgery: A comparison between intra-articular ropivacaine and levobupivacaine: A prospective, double-blinded, randomized controlled study. Saudi J Anaesth. 2014;8(3):368-73. https://doi.org/10.4103/1658-354x.136435

3. Rautoma P, Santanen U, Avela R, Diclofenac premedication but not intra-articular ropivacaine alleviates pain following day-case knee arthroscopy. Can J Anaesth.

2000;47(3):220-4.

https://doi.org/10.1007/bf03018916

4. Fowler SJ, Symons J, Sabato S, et al. Epidural analgesia compared with peripheral nerve blockade after major knee surgery: a systematic review and meta-analysis of randomized trials. Br J Anaesth.

2008;100(2):154-64.

https://doi.org/10.1093/bja/aem373

5. Richman JM, Wu CL. Epidural analgesia for postoperative pain. Anesthesiol Clin North America. 2005 ;23(1):125-40. https://doi.org/10.1016/j.atc.2004.11.004

6. Abdallah FW, Whelan DB, Chan VW, et al. Adductor Canal Block Provides Noninferior Analgesia and Superior Quadriceps Strength Compared with Femoral Nerve Block in Anterior Cruciate Ligament Reconstruction. Anesthesiology. 2016;124(5):1053-

64.

https://doi.org/10.1097/aln.000000000000 $\underline{1045}$

7. Grape S, Kirkham KR, Baeriswyl M, et al. Analgesic efficacy of sciatic nerve block in addition to femoral nerve block in patients undergoing total knee arthroplasty: a systematic review and meta-analysis. Anaesthesia.

2016;71(10):1198-209.

https://doi.org/10.1111/anae.13568

8. Roy R, Agarwal G, Pradhan C, et al. Ultrasound guided 4 in 1 block - a newer, single injection technique for complete postoperative analgesia for knee and below knee surgeries. Anaesth Pain \& Intensive
Care $2018 ; 22(1): 87$

https://doi.org/10.4103/0019-5049.186027

9. Vishvanatha S, Kallapa S. Continuous

Femoral Nerve Blockade versus Epidural

Analgesia for Postoperative Pain Relief in

Knee Surgeries: A Randomized Controlled Study Anaesthesia. 2017; 11(3): 599-605. https://doi.org/10.4103/0259-1162.206852

10. Abdallah FW, Whelan DB, Chan VW, et al. Adductor Canal Block Provides Noninferior Analgesia and Superior Quadriceps Strength Compared with Femoral Nerve Block in Anterior Cruciate Ligament Reconstruction. Anesthesiology. 2016;124(5):1053-64. https://doi.org/10.1097/aln.000000000000104 $\underline{5}$ 